\title{
DOES INTEGRATED CONSERVATION OF TERRESTRIAL ORCHIDS WORK?
}

\author{
Nigel D. Swarts ${ }^{1,2,5}$, ANDRew L. BatTy ${ }^{1,2}$, StePhen HopPeR ${ }^{3,4}$ \\ \& KingSLEY W. DiXON ${ }^{1,4}$ \\ ${ }^{1}$ Kings Park and Botanic Garden, Fraser Ave, West Perth, 6005, Western Australia \\ ${ }^{2}$ School of Earth and Geographical Sciences, Faculty of Natural and Agricultural Science, \\ University of Western Australia, Nedlands, 6009, Western Australia \\ ${ }^{3}$ Royal Botanic Gardens, Kew, Richmond, Surrey TW9 3AB, UK \\ ${ }^{4}$ School of Plant Biology, University of Western Australia, Nedlands, 6009, Western Australia \\ ${ }^{5}$ Author for correspondence: nswarts@bgpa.wa.gov.au
}

KEY WORDS: terrestrial orchids, integrated conservation, microsatellites, mycorrhiza, pollination

\section{Introduction to integrated conservation}

Effective plant conservation involves careful consideration and difficult choices when investing limited resources to conservation programs and policies. The conservation practice must integrate the understanding of existing and future environmental threats, taxonomic distinctiveness, numbers of individuals in populations, reproductive biology, ex situ propagation and the maintenance of evolutionary processes influencing population distribution patterns. For this to be possible, conservation should involve detailed experimentation directed at continued survival of the species in both an on site (in situ) ecological context and off site (ex situ) laboratory based context (Ramsay \& Dixon, 2003). Thus the development of effective conservation strategies, must strike a balance between the need for urgent action to avoid further loss and the search for essential information and understanding of the species or ecosystem to be conserved. The integrated conservation strategy emphasizes the study of interactions among land conservation, biological management, ex situ research and propagation and (re)introduction and habitat restoration (Hopper, 1997). Integrated conservation approaches vary according to different species with their habitats and distribution characteristics; however the basic concept remains the same (Fig 1).

\section{Orchid Conservation}

Many orchids are characterised by a symbiotic relationship with a mycorrhizal fungus and variety of pollination syndromes (Le Tacon \& Selosse 1994, Rasmussen 1995). The interaction of these attributes with often specialised habitat requirements has played a significant role in the evolutionary diversification and the present distribution of these flowering plants (Cozzolino \& Wedmer 2005, Otero \& Flanagan 2006).

To recover a rare or threatened orchid species in a timely and effective manner, an integrated conservation approach must be applied to the understanding of plant-fungus interactions, pollination syndromes, population genetic structure and evolutionary processes, in situ habitat requirements and ex situ conservation concepts. Many workers have effective-

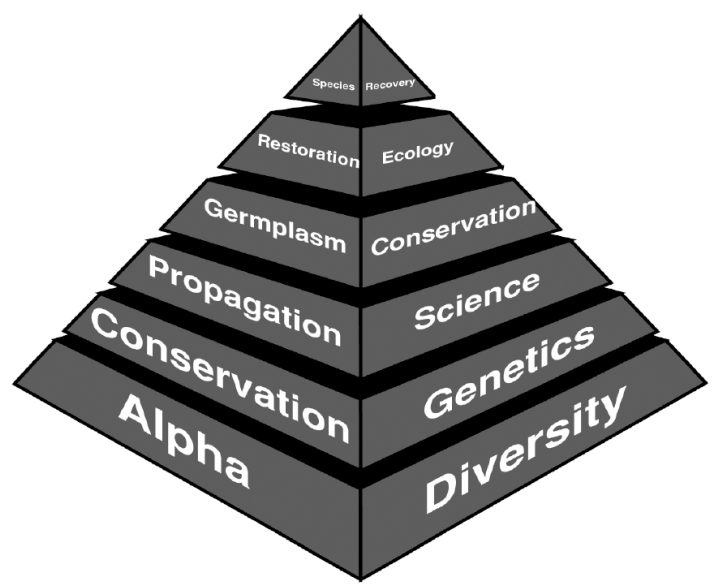

FiguRE 1. A pyramid model of the integrated conservation concept (Dixon \& Batty 2003). Species recovery (top step) is an integrated process requiring all the five lower steps shown in this figure. 
ly researched these principles as singular entities in a conservation context, however, over the last decade with the successful development of a raft of new conservation technologies can the integrated approach be adopted at a whole species level. The aim of this study is to test the principle of a science-based integrated conservation approach (Falk 1990) towards the recovery of nationally endangered orchid Caladenia huegelii H. G. Reichb. Key elements of the study include understanding the basis of rarity in the species, defining and abating threatening processes and developing translocation and management strategies to enhance species resilience and persistence in extant locations.

Caladenia huegelii occurs from Perth to Busselton in Western Australia along the Bassendean Sand geological system characterized by deep, highly infertile siliceous sands in species rich banksias woodland (Hoffman \& Brown 1992). Extensive surveying of the all known populations and potential habitat for new populations over three successive growing seasons revealed that the species is significantly reduced to just 18 locations, seven of these are new populations and four of the 18 contain just one plant. Urban development contributed to the loss of populations and largescale loss of suitable habitat for recruitment opportunities. Grazing of leaves and buds by caterpillars, tuber diggings by bandicoots and the grazing of developing seed capsules by kangaroos were observed as other threatening processes and key management issues.

\section{Population Genetics}

Conservation strategies for the maintenance of genetic diversity rely greatly on detailed study of the genetic structure, demographic history and evolutionary potential of plant populations (Fay \& Krauss 2003). To develop an understanding of the genetic structure and variability of $C$. huegelii populations, microsatellite markers were used to screen 460 samples representing all populations over its geographical range for polymorphism across seven loci. Genetic divergence among populations was correlated with geographical distance investigating genetic isolation on both population and regional levels. In results comparable to another sexually deceptive Caladenia species, C. huegelii exhibited similar low levels of genetic differentiation (Fst $=0.102$ ), with $90 \%$ of genetic variation partitioned within populations (Peakall \& Beattie 1996).

\section{Fungal Associations}

Fungal associates of Caladenia species show a distinctively fragmented distribution in the landscape, a feature that may be contributing to the rarity of these orchids (Batty et al. 2001a, Brundrett et al. 2003). Working with $C$. huegelii and common sympatric congeners, we investigated the diversity and specificity of associated endophytes isolated from adult plants over the geographical range of the orchid through large scale in situ and in vitro matrix germination experiments. These studies demonstrated a highly specific plant-endophyte relationship in $C$. huegelii indicating that orchid distribution may be limited to the type and availability of a specific symbiont at a site, a possible driver of rarity in this species. Endophytes isolated from $C$. huegelii adult plants over the range of the taxon and germinated in situ protcorms were found to effectively germinate seed from across the habitat range of the orchid. No significant home site advantage was observed for orchid seed germinating on an endophyte collected from the same plant. Common congeneric species utilized a range of available endophytes in both in situ and in vitro germination trials including the $C$. huegelii fungus suggesting potential implications for competitive niche occupancy.

The results of high specificity of $C$. huegelii endophytes were reinforced by sequencing the highly variable ITS region of 50 endophytes isolated from both adult plants of $C$. huegelii and in situ protocorms, using the fungal specific primers ITS1 and ITS4, finding identical sequences of all endophytes sequenced. Further sequencing of endophytes isolated from common congeners revealed sequence variation when analyzed with those from $C$. huegelii providing additional support to germination results. Analysis of the ITS sequences isolated from the range of Caladenia species showed high similarities with a sequence from a Sebacina vermifera originally isolated from Caladenia dilatata (Bougoure et al. 2005) and matching sexual stages identified by Warcup (1971) for Caladenia. 


\section{Pollination Syndrome}

This species adopts a sexually deceptive pollination syndrome employing male thynnid wasps for the transfer of pollen from one plant to another (Stoutamire 1983). We investigated the natural abundance of the specialized wasp pollinators at the key study site at Ken Hurst Park site using the established 'baiting' techniques used in sexual deceptive systems. A detailed study of the natural pollination success for C. huegelii showed for the species a low rate of seed set with $<4 \%$ of flowering plants producing seed in both the 2005 and 2006 seasons. The study compared the success to other sexually deceptive pollinated orchids and showed that $C$. huegelii is on the lower end of the pollination success scale compared with related genera such as Drakaea where pollination success rates can be in excess of $80 \%$ (R. Phillips, pers. comm. 2006) and was very low compared to most other sexually deceptive orchids with a mean pollination rate of 33.1\% (Tremblay et al. 2005, J. See, pers. comm). All baiting trials indicated an absence of the pollinator at the site based on the sampling method used. Comparison with pollination success in other Caladenia species showed the low levels of pollination recorded in C. huegelii fall within the pollination range for Caladenia indicating that the low seed set in C. huegelii is not unexpected. Clearly to sustain suitable seed output from wild and reintroduced populations of the orchid, there may be either the need to increase pollinator abundance or have intervention management involving artificial pollination.

\section{Reintroduction and transplanting}

Protocorms generated from germination experiments were used in subsequent pot and field trials to optimize tuberisation in developing seedlings thus increasing the transfer success of plants in situ. The reintroduction of orchids to field sites was markedly enhanced by outplanting two-year old seedlings over one-year old seedlings with irrigation and protective cages enhancing plant growth and overall survival. Out-plants were monitored fortnightly over the 2006 growing season and $100 \%$ survival was observed for two-year old seedlings compared to $5 \%$ survival in the seedlings out-planted in their first season of growth. Increased survival and seedling growth was observed in seedlings outplanted in close proximity to adult plants in comparison with artificially inoculated substrate and the no inoculum control further reinforcing endophytic potential for reintroduction success.

\section{Ex situ conservation}

Although in situ conservation of threatened taxa takes precedence in management priorities, the requirement for ex situ conservation and storage of germplasm is increasingly recognized as an imperative tool in the preservation and maintenance of biodiversity (Batty et al. 2001b, Fay \& Kruass, 2003). Using the results of the molecular study to determine significant populations and the methods of Batty et al (2001b) to ensure long term survival of stored germplasm, a representative sample of viable $C$. huegelii seed was placed in liquid nitrogen $\left(-196^{\circ} \mathrm{C}\right)$. Although no significant sequence variation of $C$. huegelii associated endophytes was observed, a range of isolates that best promoted seed germination across all populations have also been successfully preserved in liquid nitrogen.

\section{Conclusion}

This study has demonstrated that within a relatively short time frame it is possible to develop a comprehensive and focused data set leading to effective integrated conservation. Using seedling establishment in the wild as a key benchmark of success the study results provided confidence that recovery operations can be effectively undertaken if there is an understanding of:

- Mycorrhizal specificity

- Biogeographic variation in mycorrhiza

- Genetic diversity of plants and fungi

- Seed banking and endophyte banking

- Pollination ecology.

The challenge now is to adopt and test these key principles in terms of multi-species recovery operations for orchids possibly starting with congeneric taxa and then working on phylogenetically related taxa. The future of orchid conservation depends on effective and timely delivery of orchid conservation where there are active links between on-site conservation actions and research programs. 


\section{LITERATURE CITED}

Batty A.L., K.W. Dixon, M. Brundrett \& K. Sivasitthampara. 2001a. Long-term storage of mycorrhizal fungi and seed as a tool for the conservation of endangered Western Australian terrestrial orchids. Austral. J. Bot. 49: 1 - 10.

Batty A.L., K.W. Dixon, M. Brundrett \& K. Sivasitthampara. 2001b. Constraints to symbiotic germination of terrestrial orchid seed in a Mediterranean bushland. New Phytol. 152: 511 - 520.

Bougoure J.J., Bougoure D.S., Cainey J.W.G. \& Dearnaley J.D.W. (2005) ITS - RFLP and sequence analysis of endophytes from Acianthus, Caladenia and Pterostylis (Orchidaceae) in southeastern Queensland. Mycol. Res. 109(4): $452-460$.

Brundrett M.,A. Scade, A.L. Batty, K. Dixon \& K. Sivasithampara. 2003. Development of in situ and ex situ seed baiting techniques to detect mycorrhizal fungi from terrestrial orchid habitats. Mycol. Res. 107: 1210-1220.

Cozzolino S. \& A. Widmer. 2005. Orchid diversity: an evolutionary consequence of deception? Trends Ecol. Evol. 20: 487-494.

Dixon K.W. \& A.L. Batty. 2003. Integrated conservation of Australian orchids - a local and global perspective. In 'Orchid Conservation Forum II'. Royal Botanic Gardens, Melbourne.

Falk D.A. 1990. Integrated strategies for conserving plant genetic diversity. Ann. Missouri Bot. Gard. 77: 38-47.

Fay M.F. \& S.L. Krauss. 2003. Orchid conservation genetics in the molecular age. Pp. 91-112 in: K.W. Dixon, S.P. Kell, R.L. Barrett \& P.J. Cribb (eds.). Orchid Conservation. Natural History Publications (Borneo): Kota Kinabalu, Sabah, Malasia.
Hoffman N. \& A. Brown. 1992. Orchids of South-West Australia, 2nd Edition. University of Western Australia Press, Nedlands.

Hopper S.D. 1997. An Australian perspective on plant conservation biology in practice. Pp. 255-278 in: P.L. Fiedler \& P.M Kareiva (eds.). Conservation Biology for the Coming Decade. Chapman and Hall: New York.

Le Tacon F. \& M.A. Selosse 1994) The mycorrhizal symbiosis in the evolution and the colonization of continents by the life. [French]. Acta Bot. Gallica 141: 405419.

Otero J.T. \& N.S. Flanagan. 2006. Orchid diversity beyond deception. Trends Ecol. Evol. 21 : 64-65.

Ramsay M.M. \& K.W. Dixon. 2003. Propagation science, recovery and translocation of terrestrial orchids. Pp. 259-288 in: K.W. Dixon, S.P. Kell, R.L. Barrett \& P.J. Cribb (eds.). Orchid Conservation. Natural History Publications (Borneo): Kota Kinabalu, Sabah, Malasia.

Rasmussen H.N. 1995. Terrestrial Orchids, from Seed to Mycotrophic Plant. Cambridge University Press: Cambridge.

Stoutamire W.P. 1983. Wasp-pollinated species of Caladenia (Orchidaceae) in South-western Australia. Austral. J. Bot. 31: 383-94.

Tremblay R.L., J.D. Ackerman, J.K. Zimmerman \& R.N. Calvo. 2005. Variation in sexual reproduction in orchids and its evolutionary consequences: a spasmodic journey to diversification. Bot. J. Linn. Soc. 84: 1-54.

Warcup J.H. 1971. Specificity of mycorrhizal association in some Australian terrestrial orchids. New Phytol. 70: 41-46.

Nigel Swarts is currently writing his $\mathrm{PhD}$ thesis on the integrated conservation of rare and threatened terrestrial orchids with the University of Western Australia. He is based and supported by Kings Park and Botanic Gardens in Western Australia and forms part of their orchid conservation group. He has a particular interest in threatened orchids of Australia's southwest with special attention given to the Caladenia genus

Kingsley Dixon has over 20 years experience in researching the ecology and physiology of Australian native plants and ecosystems. He leads a science group comprising botanical and restoration sciences and, as Director of Science at the Botanic Gardens and Parks Authority (BGPA), has developed a strong multi-disciplinary approach to conservation and restoration of native plant biodiversity and degraded landscapes. This research group has contributed significantly to seed science in Australia, with major advances in understanding seed dormancy as well as orchid seed conservation.

Stephen Hopper is director of the Royal Botanic Gardens, Kew. He has worked on Australian orchid systematics and conservation since 1973. Current interests include generic classification of Australian orchids, and the evolution of southwest Australian orchids. 